\title{
Cancer survival differences between South Asians and non-South Asians of England in 1986-2004, accounting for age at diagnosis and deprivation
}

\author{
C Maringe ${ }^{\star}, 1, \mathrm{R} \mathrm{Li}^{1}, \mathrm{P}$ Mangtani ${ }^{1}, \mathrm{M} \mathrm{P}$ Coleman $^{1}$ and B Rachet ${ }^{1}$ \\ ${ }^{1}$ Faculty of Epidemiology and population Health, London school of Hygiene and Tropical Medicine, Keppel Street, London WC1E \\ 7HT, UK
}

Background: South Asian migrants show lower cancer incidence than their host population in England for most major cancers. We seek to study the ethnic differences in survival from cancer.

Methods: We described and modelled the effect of ethnicity, time, age and deprivation on survival for the five most incident cancers in each sex in South Asians in England between 1986 and 2004 using national cancer registry data. South Asian ethnicity was flagged using the validated name-recognition algorithm SANGRA (South Asian Names and Group Recognition Algorithm).

Results: We observed survival advantage in South Asians in earlier periods. This ethnic gap either remained constant or narrowed over time. By 2004, age-standardised net survival was comparable for all cancers except three in men, where South Asians had higher survival 5 years after diagnosis: colorectal (58.9\% vs 53.6\%), liver (15.0\% vs 9.4\%) and lung (15.9\% vs $9.3 \%)$. Compared with non-South Asians, South Asians experienced a slower increase in breast and prostate cancer survival, both cancers associated with either a screening programme or an early diagnosis test. We did not find differential patterns in survival by deprivation between both ethnicities.

Conclusions: Considering recent survival trends, appropriate action is required to avoid deficits in cancer survival among South Asians in the near future.

People of Indian, Pakistani and Bangladeshi origin (South Asian) comprise the biggest minority ethnic group in the UK, constituting $4 \%$ of the total UK population, or half of all the non-European population in the UK (Office for National Statistics, 2003). South Asians mostly migrated to the UK in the 1950s and 1960s. The literature of cancer patterns in migrant populations refer to them as either South Asians or South Asian migrants, often without distinguishing between the first, second and the third generations. Patterns of cancer incidence, mortality and survival in South Asians in the UK have been shown to differ from the host population (Moles et al, 2007; Ali et al, 2010; Mangtani et al, 2010; Maringe et al, 2013). Incidence from the common cancers such as breast, prostate, lung and colorectal are generally lower in South Asians, though the rates are steadily converging (Winter et al, 1999; Smith et al, 2003; Maringe et al, 2013).

Previous national and regional studies of breast cancer survival have shown higher survival for South Asians compared with the non-South Asian population, even though they tend to live in poorer areas (dos Santos Silva et al, 2003; Farooq and Coleman, 2005; Jack et al, 2009). Survival from oral (Warnakulasuriya et al, 1999; Moles et al, 2007) and cervical cancers (Bates et al, 2008) was higher among South Asian migrants compared with non-South Asians. By contrast, a regional study of cancer survival in children and young adults under 30 years of age showed that differences

*Correspondence: C Maringe; E-mail: Camille.Maringe@lshtm.ac.uk

Received 19 November 2014; revised 14 April 2015; accepted 22 April 2015;

published online 16 June 2015

(c) 2015 Cancer Research UK. All rights reserved 0007-0920/15

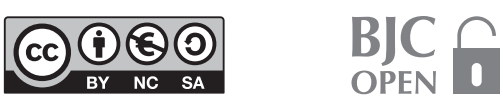


between South Asians and non-South Asians vary with the type of tumour: South Asians had poorer survival from leukaemia and lymphoma compared with non-South Asians, but better outcomes from solid tumours (van Laar et al, 2012).

We sought to determine how cancer survival in the South Asian population of England has changed between 1986 and 2004, how it varies by age and deprivation, and how these patterns compare with those observed in non-South Asians. We used national cancer registry data, focusing on the five most common cancers in South Asians in the period 2000-2004 (Maringe et al, 2013): prostate, lung, colorectal, non-Hodgkin lymphoma (NHL) and liver in men, and breast, uterus, ovary, colorectal and NHL in women (index cancer). Deaths in older South Asians are not always well captured, possibly due to more frequent travel to South Asia once retired, which could compromise the estimation of the survival. We therefore limited the analysis to patients aged 15-69 years at diagnosis.

\section{MATERIALS AND METHODS}

Information on ethnic origin in the national cancer registry and population data were incomplete. Therefore, we applied the validated SANGRA (South Asian Names and Group Recognition Algorithm) algorithm (Maringe et al, 2013), after ethical and statutory approvals, to flag all South Asian patients in the national cancer registry, making use of their distinctive names. Despite already high sensitivity and specificity (Nanchahal et al, 2001) against self-assigned ethnicity (considered as the gold standard), we also visually inspected all names selected as South Asians to increase the specificity of the algorithm (Mangtani et al, 2010).

A total of 1050644 people were diagnosed in England between ages 15 and 69 years with an invasive, primary malignancy of interest. They were registered in the national cancer registry and followed up for their vital status until 31 December 2007. The quality of the data were assessed using standard criteria (Li et al, 2014), leaving 997104 patients (94.9\%) for analysis, among whom 13979 (1.4\%) were South Asians (Table 1). Proportions of exclusion varied widely across cancers and between ethnicities: overall 9.1\% in South Asians and 5.0\% in non-South Asians, but reaching $11.2 \%, 14.5 \%$ and $21.9 \%$ for NHL, lung and liver cancers in South Asians, respectively, that is, around 5-6\% higher than in nonSouth Asians. South Asians were more likely than non-South Asians to be excluded because of unknown vital status (5.3\% vs $0.9 \%$ ), but the proportion of death-certificate-only registrations was generally similar or slightly higher among non-South Asians. Patients with no information on the postcode of residence at the time of diagnosis could not be assigned to an ecological deprivation score so they were also excluded (3058, 0.15\%, similar for both ethnic groups).

Net survival, the metric of interest, is the survival that the cancer patients would experience after adjustment for death from other causes. Net survival is the only measure enabling comparison of cancer survival between populations experiencing different levels of mortality from other causes, as seen between South Asians and non-South Asians (e.g., for cardiovascular disease). In the absence of reliable information on the cause of death, net survival is estimated by comparing the overall mortality of the cancer patients with the mortality that would have been expected if they did not have cancer ('expected' or 'background' mortality), using a nonparametric estimator (Pohar Perme et al, 2012).

Expected mortality was derived from population life tables that we constructed by single year of age (0-99 years) and single calendar year (1986-2004), sex and deprivation category, for the entire population of England, as well as separately for the South Asian population. Ethnicity is not recorded at death, and death rates for South Asians were constructed by assigning ethnicity from the name in the national mortality database in the Office for National Statistics
(Mangtani et al, 2010), in the same way as for the cases in the national cancer registry. The number of deaths was taken as the mean annual number of deaths in the 3 years centred on the census year, to reduce the effect of random fluctuation. Populations were taken from the censuses for 1991 and 2001. Each small area was mapped to a deprivation index: the Carstairs index in 1991 (Carstairs, 1995) and the income domain of the Indices of Multiple Deprivation (IMD 2004) in 2001 (Department of the Environment Transport and the Regions, 2000), and aggregated into five categories by quintile of the national distribution. All-cause death rates per 100000 per year by deprivation were then derived after aggregation of the number of deaths (numerator) and the population (denominator) by age, sex and ethnic group for each small area in England (Enumeration District in 1991, Lower Super Output Area in 2001) into the five deprivation categories. Linear interpolation was used to obtain a life table for each calendar year between 1991 and 2001. The 2001 life tables for South Asians and non-South Asians were assumed to apply over the years 2002-2004, and the 1991 life tables over the years 1986-1990. This enabled us to build a complete series of deprivation-specific life tables for South Asians and non-South Asians over the period 1986-2004. To our knowledge this is the first time that life tables specific for the South Asian population have been constructed in the UK (Cancer Research UK Cancer Survival Group, 2004).

For each cancer, we estimated net survival up to 5 years by ethnic group, age group and period of diagnosis (1986-1995, 1996-2004) using the Pohar Perme estimator (Pohar Perme et al, 2012) implemented in the STATA (StataCorp, 2013) command stns (Clerc-Urmès et al, 2014). Overall survival estimates were age standardised using the International Cancer Survival Standard weights (Corazziari et al, 2004).

Modelling the excess hazard of death enables investigating further the differences in net survival between South Asians and non-South Asians, and in particular, whether the effect of ethnicity varied with time since diagnosis, year of diagnosis, age at diagnosis and deprivation. The excess hazard of death (or excess mortality) is a measure of the mortality due to cancer over and above the expected mortality, defined earlier. In contrast with survival, a cumulative measure, the hazard is an instantaneous measure and therefore detects any changes in the rate at which excess or cancerrelated deaths occur. We modelled the excess mortality on the log-cumulative excess hazard scale, using flexible functions (Nelson et al, 2007; Lambert and Royston, 2009). We allowed for non-linear effects of age and year of diagnosis by constructing restricted cubic splines for these variables, with knots located at the tertiles of the distributions. We also tested and allowed for time-dependent effects of ethnicity and other covariates. We used the log-likelihood ratio test to compare models with and without interactions between all co-variables and ethnicity, and with and without time-dependent effects of the interactions. All analyses were conducted using the stpm2 command (Lambert and Royston, 2009) in STATA 13.

\section{RESULTS}

Patterns and trends in cancer survival. Age-standardised net survival was on average higher in South Asians than in non-South Asians, for most cancers. That contrast was stronger in 1986-1995 (Table 2). Survival was only lower in South Asians compared with non-South Asians for uterine cancer and NHL in 1986-1995. In 1996-2004, the survival advantage in South Asians was no longer seen for breast and prostate cancers especially at 5 years after diagnosis. The South Asian advantage in survival also considerably reduced between both periods for the other cancers. By 2004, survival was higher in South Asians compared with non-South Asians only for cancers of the colorectum (men), liver and lung. 
Table 1. Number of patients eligible and included for analysis, and percentage excluded, for South Asian and non-South Asian adults (15-69 years at diagnosis) diagnosed in 1986-2004 and followed up to 2007

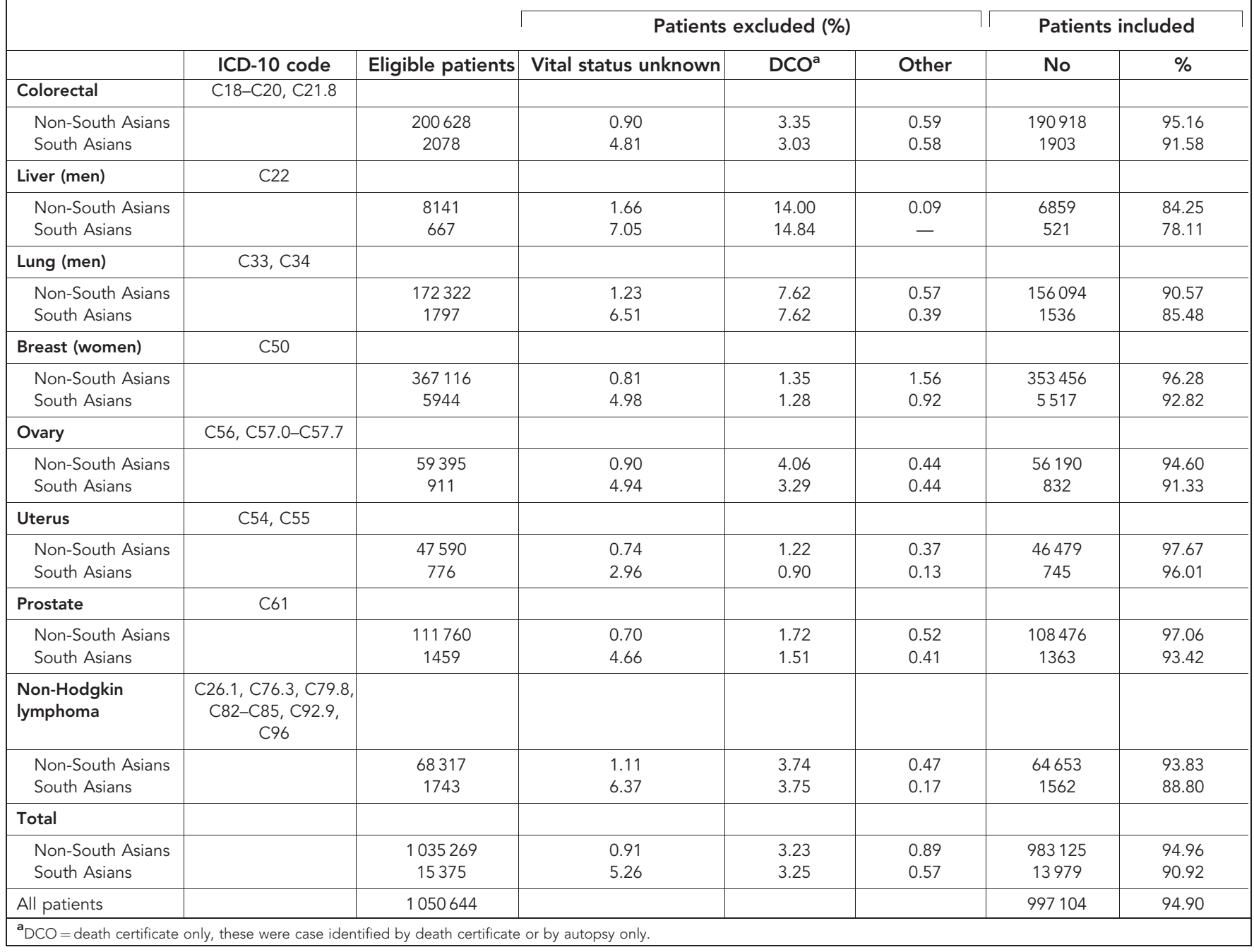

For example, in 1996-2004, 5-year colorectal cancer survival in men was 58.9\% (95\% CI 55.0-62.8) in South Asians and 53.6\% (53.1-54.1) in non-South Asians. By contrast, 1-year survival from NHL in women was $73.7 \%(68.0-79.3)$ in South Asians and $80.5 \%$ (79.8-81.1) in non-South Asians in 1996-2004; this ethnic difference in survival has reduced over time.

The reduction in the gap in survival between South Asians and non-South Asians is a result of survival in non-South Asians increasing more rapidly than among South Asians between the early and late 1990s. This general pattern was not observed for the ovarian and uterine cancer; neither was it seen for NHL for which survival improved more rapidly among South Asians.

Only lung cancer patients aged 45-54 and 55-64 years at diagnosis, and NHL patients aged 65-69 years showed evidence of differences in survival between ethnic groups in the period 1996-2004 (Supplementary Table 1).

In 1986-1995, net survival from lung cancer in South Asian men was as high as $40.9 \%$ and $26.0 \%$ at 1 and 5 years, respectively, compared with only $26.3 \%$ and $8.5 \%$ in non-South Asians. In 1996-2004, 1- and 5-year survival in South Asians apparently declined by $6 \%$ and $10 \%$, respectively, to reach $35.2 \%$ at 1 year and $15.9 \%$ at 5 years. By contrast, lung cancer survival among nonSouth Asians rose slightly, but was still lower than in South Asians (Table 2).

A very steep increase was observed in 5-year survival from prostate cancer in both ethnic groups: $20 \%$ in South Asians between $58.4 \%(43.3-73.5)$ and $78.4 \%(70.1-86.6)$ and a staggering
$30 \%$ in non-South Asians between 51.0\% (49.4-52.7) and $81.1 \%$ (80.1-82.2).

Patterns of changes in excess mortality. The excess hazard of death varied between ethnic groups for all cancers except ovarian and uterine cancers ( $P$-values 0.13 and 0.15 , respectively; Supplementary Table 2). We then tested whether the effect of ethnicity on the excess hazard changed with time since diagnosis, year and age of diagnosis and deprivation (Supplementary Table 2).

Excess cancer mortality among South Asians. South Asians experienced the usual patterns of high excess hazard in the first few months after diagnosis, followed by excess hazard close to zero at around 5 years after diagnosis (Figure 1). The excess hazard varied widely by age, and to a lesser extent by deprivation, in South Asians diagnosed with NHL, lung, liver and ovarian cancers. Excess hazard of dying in patients aged 65 years remained higher than patients aged 50 years until 5 years after diagnosis.

South Asians often experienced lower excess hazard of death than non-South Asians (lower part of Figure 1) in the 5 years that followed the cancer diagnosis. For cancers of the colorectum (women), lung (men) and prostate, South Asians had lower hazard of death from cancer throughout the first 5 years after diagnosis. That advantage in excess mortality widened with time since diagnosis for lung cancer, from a 15\% lower mortality shortly after the diagnosis up to $75 \%$ at 5 years. On the other hand, in men, the excess hazard of death for colorectal and liver cancer became lower in South Asians only at least after 6 months since diagnosis, leading 
Table 2. Age-standardised 1- and 5- year net survival (\%), by cancer, sex and period of diagnosis for South Asians and non-South Asians

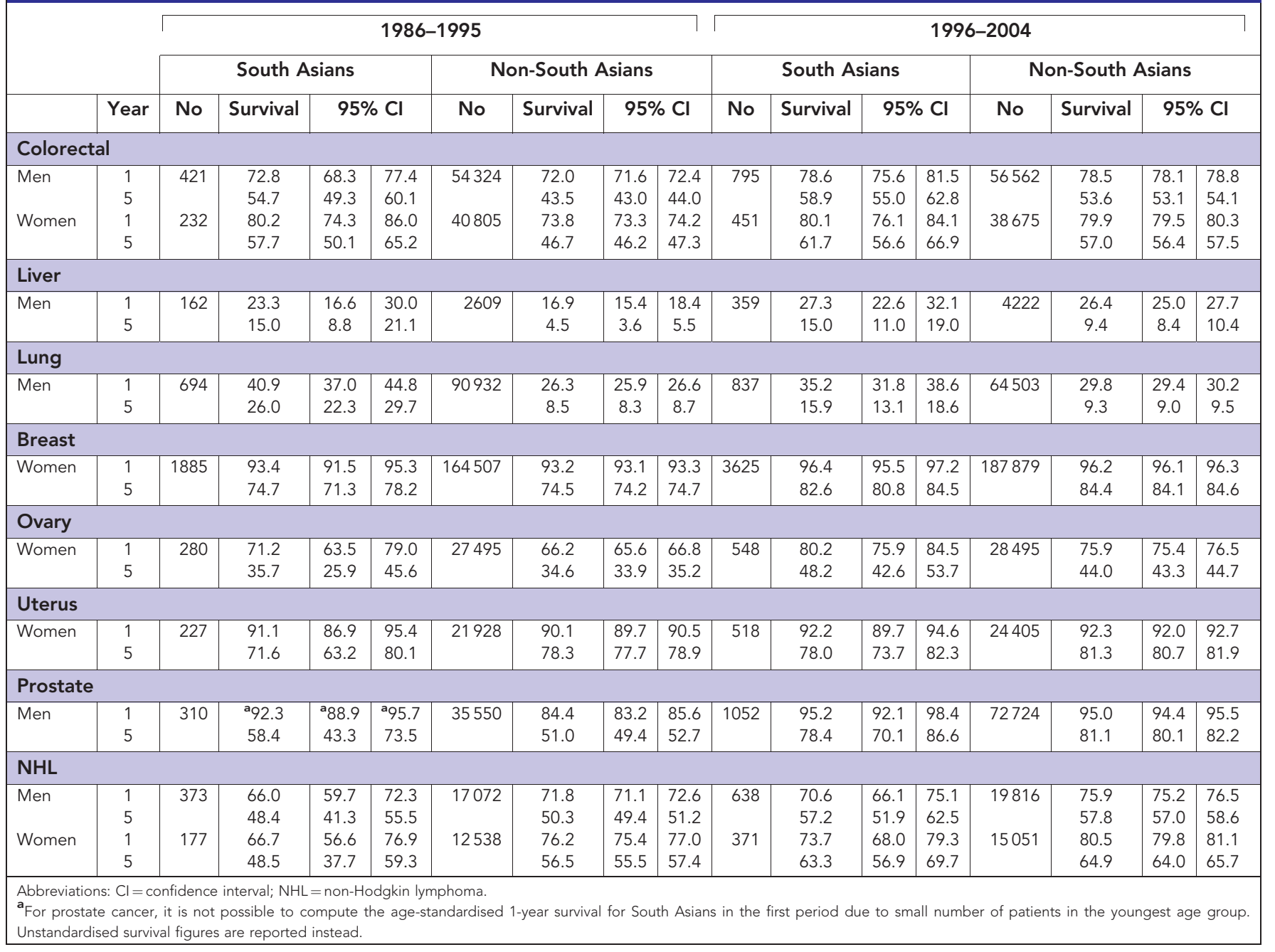

to $50 \%$ and $75 \%$ reduction at 5 years, respectively. For ovarian cancer, lower excess mortality was seen in South Asians only until 6 months after diagnosis.

By contrast, South Asians experienced a higher excess mortality than non-South Asians for NHL (EHR from 1.23 (1.07-1.42) and 1.39 (1.14-1.71) for men and women, respectively) and for cancers of the breast (EHR: 1.06 (1.00-1.12)) and the uterus (EHR: 1.14 $(0.96-1.34))$. This deficit in NHL survival vanished after 18 or 36 months since diagnosis for men and women, respectively.

Temporal trend in excess hazard of death by ethnicity. Among the few cancers for which the effect of ethnicity seemed to vary with year of diagnosis (Supplementary Table 2), 3-month and 1 -year excess mortality from colorectum and liver cancer decreased rapidly in non-South Asians between 1986 and 2004, whereas it remained constant in South Asians, resulting in a reduced or null gap in excess mortality between both ethnic groups (Figure 2). Trends in 5-year excess mortality were less clear. For lung cancer (men), the trends in excess mortality at 3 months and 1 year diverged between ethnic groups, with a patent increase in South Asians and a slight decrease in non-South Asians: in 2004, excess hazards in non-South Asians were at 0.98 (0.96-1.00) and 0.67 $(0.66-0.69)$ at 3 months and 1 year after diagnosis, respectively, similar to the excess hazards in South Asians (0.94 (0.84-1.05) and $0.55(0.50-0.62)$ at 3 months and 1 year). The excess hazards for prostate cancer decreased much more quickly in non-South Asians than South Asians, at all times after diagnosis.
Age pattern in excess hazard of death by ethnicity. Excess mortality by ethnicity varied differently according to age at diagnosis for colorectal (women), NHL (men) and uterine cancer patients (Figure 3 and Supplementary Table 2). In South Asians, patients aged 15-35 years at diagnosis tended to die more from their colorectal cancer than the patients aged 50-69 years, while in non-South Asians, the age pattern was reversed, regardless of time since diagnosis. For the uterine cancer, a general U-shape of the excess mortality was observed for both ethnicities, but mirrored with the highest mortality among the younger patients for the non-South Asians and among the older patients for the South Asians: at 3 months after diagnosis, the excess hazards were 0.15 (95\% CI 0.11-0.21) in South Asians aged 15 years and 0.17 (95\% CI $0.13-0.23)$ in non-South Asians aged 69 years. For NHL male cancer patients, the increasing excess mortality with increasing age was much more marked in South Asians than among non-South Asians, especially 3 months after diagnosis.

Deprivation pattern in excess hazard of death by ethnicity. Deprivation patterns in South Asians were similar to that in nonSouth Asians for most cancers (Supplementary Table 2) with greater excess mortality in more deprived population. The trends in breast cancer excess mortality by deprivation was slightly steeper in South Asians than non-South Asians, while, for prostate cancer, the high excess mortality observed in non-South Asians shortly after the diagnosis was attenuated in South Asians for all deprivation categories (Figure 4). NHL cancer showed very 

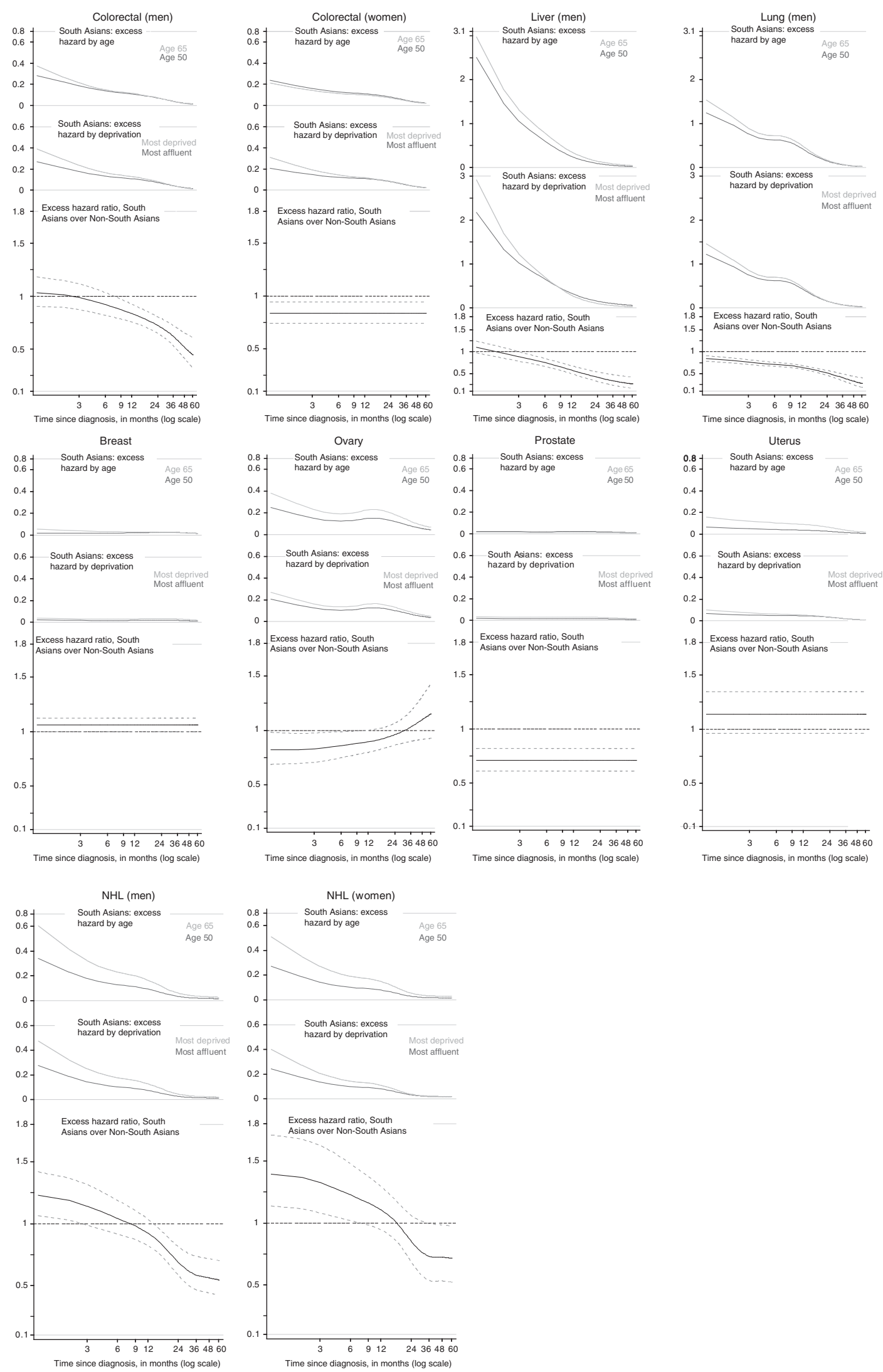

Figure 1. Excess hazard of death by age and deprivation in South Asians for patients diagnosed in 2004, and excess hazard ratios comparing South Asians to non-South Asians. 

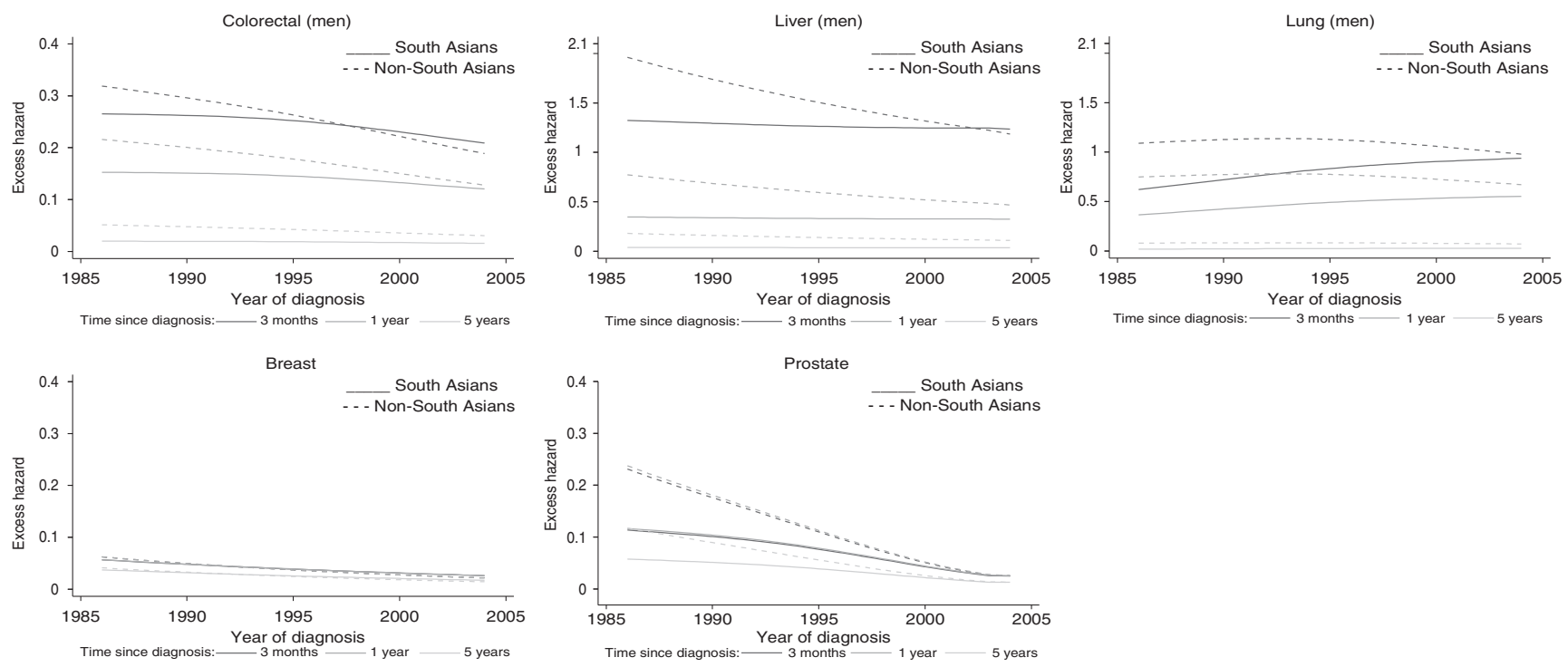

Figure 2. Change in excess hazard with year of diagnosis, by ethnic group at 3,12 and 60 months after diagnosis, for cancers selected based on the significance of the interaction term year of diagnosis $\times$ ethnicity (patients aged 50 , most affluent).
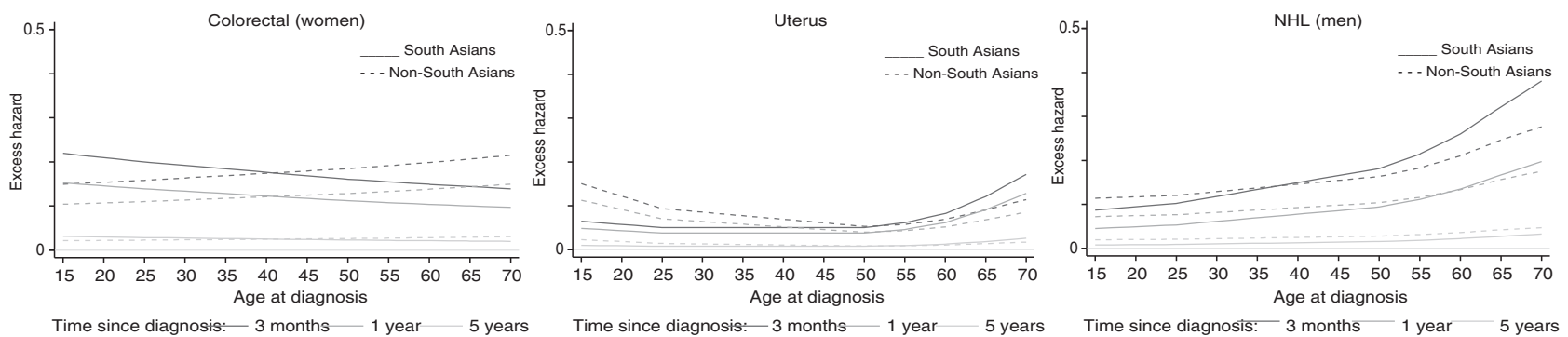

Figure 3. Change in excess hazard with age at diagnosis, by ethnic group at 3,12 and 60 months after diagnosis, for cancers selected based on the significance of the interaction term age at diagnosis $\times$ ethnicity $(2004$, most affluent).

unstable excess hazard estimates by deprivation for South Asians, leading to a triangular relationship between excess mortality and deprivation: most probably, it was the reason for an interaction between ethnicity and deprivation.

\section{DISCUSSION}

This study aims at measuring trends in survival for the five most common cancers in each sex in South Asians living in England. To our knowledge, this is the first time survival from cancers other than breast cancer has been estimated for this ethnic group for the whole of England and over such a long period of time.

In the late 1980s, net survival in South Asians was either higher (colorectum, liver, lung and prostate) or similar to that in non-South Asians (breast, ovary, uterus and NHL). By 2004, however, the picture was very different: South Asians still experienced higher survival for lung cancer, but lower survival for NHL, while survival from other cancers had become similar in both ethnic groups. Adjusting for age at diagnosis and deprivation only resulted in small and inconsistent effects on the overall ethnic differences in survival.

We restricted our analysis to adults aged 15-69 years in order to avoid potential bias in the estimation of cancer incidence and survival among elderly South Asians. South Asians also tend to be diagnosed at younger ages than non-South Asians (Downing et al, 2011; Maringe et al, 2013).

The literature reports a mixed role for ethnicity and socioeconomic background in cancer survival (Parikh-Patel et al, 2006;
Moles et al, 2007, Jack et al, 2009, National Cancer Intelligence Network, 2009, van Laar et al, 2012, Ortiz-Ortiz et al, 2014). Ethnic differences in 1- and 3-year age-standardised survival for cancers of the colorectum, breast (women) and prostate were reported to be minor, using HES-derived ethnicity (National Cancer Intelligence Network, 2009). It was also reported (National Cancer Intelligence Network, 2009), as we do here, higher lung cancer survival for Asian patients diagnosed between 2002 and 2006. Our study, however, presents methodological differences. To estimate the net survival for each ethnic group, we constructed separate life tables of background mortality for South Asians by single year of age, sex, deprivation category and calendar year (Cancer Research UK Cancer Survival Group, 2004; available on our website at publication). The survival models we employed included age at diagnosis, year of diagnosis and deprivation status. This approach provides the tightest possible control for differences and trends in background mortality by ethnicity and deprivation.

Name recognition remains the best way to identify persons of South Asian ethnicity when studying trends in population-based cancer survival in England, because self-assigned ethnicity is not routinely recorded at registration. Moreover, because some nonSouth Asian Muslim names could be confounded with common South Asian names, we have proceeded to additional checks of the South Asian names selected by the algorithm among cancer patients. The use of a name-recognition algorithm on cancer incidence data permits the inclusion of second and subsequent generations of migrants in the analyses, unlike using the country of birth as a proxy for ethnicity. Self-assignment of ethnicity, seen as the gold standard, is still incomplete in most health data sets 

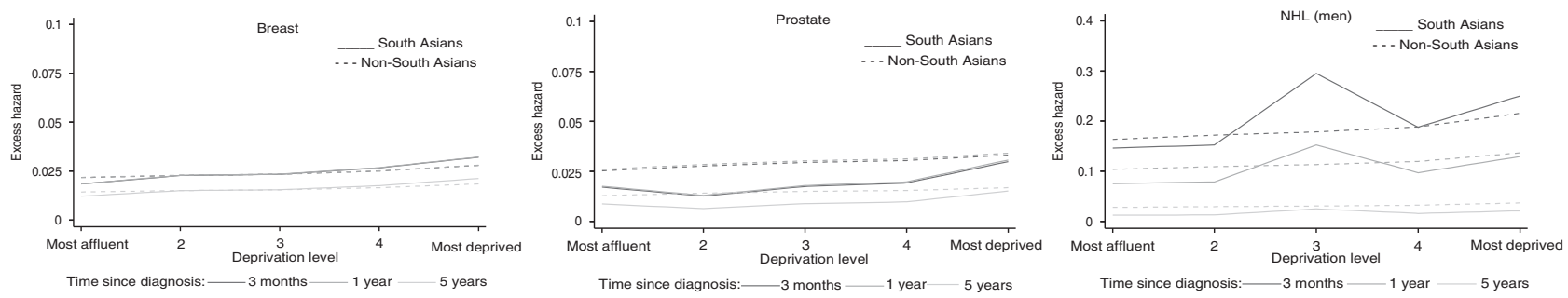

Figure 4. Change in excess hazard with deprivation, by ethnic group at 3,12 and 60 months after diagnosis, for cancers selected based on the significance of the interaction term deprivation $\times$ ethnicity $(2004$, most affluent).

(such as HES), which contain many records with two or more conflicting entries for the same patients. It has been advocated that despite their drawbacks, such data should be analysed anyway in order to drive up their quality (Aspinall and Jacobson, 2007). One study of the impact of self-assigned ethnicity on breast cancer outcomes in South Asians in the UK concluded that any technique (including imputation of missing ethnicity) used to identify the 'best' ethnicity led to very similar results (Downing et al, 2011).

Differences in cancer incidence and survival between Blacks and Whites (Bassett and Kreiger, 1986; Lannin et al, 1998; Breen et al, 1999) or within the Asian ethnic group (Pineda et al, 2001; Robbins et al, 2007, Bates et al, 2008; Gomez et al, 2010) have been reported in the US for decades, where ethnicity is sometimes used as a proxy for wealth (Ash and Robinson, 2009). In the US, where the South Asian population also comprises Japanese and Filipinos, their breast and prostate cancer survival is lower than in non-Hispanic whites (Robbins et al, 2007).

The hypothesis claiming that the ethnicity gap in survival widens with increasing amenability of cancer to treatment (expressed by increasing net survival) was tested in the US using SEER population-based data in the late nineties (Tehranifar et al, 2009). By amenability to treatment, we mean easy access to efficient treatment. Compared with the White population, the survival advantage initially found in Asian and Pacific Islanders (including South Asians) tended to disappear with increasing amenability, while deficit in survival observed in all other ethnic groups widened. Similarly, we found no significant difference between the survival experienced by South Asians and non-South Asians, during 1996-2004, for cancers with good survival and good amenability to treatment (prostate, uterine and breast cancers, 5 -year survival higher than $70 \%$ ) or for partly amenable cancers (NHL, ovary and colorectal cancer). The South Asian survival advantage remained over $5 \%$ higher in 'not amenable' cancers such as liver (at 5 years) and lung (at both 1 and 5 years). In the US, Asian ethnicity was identified as a favourable prognostic factor for overall survival from non-small cell lung cancer in California, even after adjustment for smoking status (Ou et al, 2009). We also found a significant advantage in lung cancer survival for South Asians, which matches the theory of minorities and amenability of cancers (Tehranifar et al, 2009). However, caution should be made in particular in the US where access to healthcare strongly depends on socioeconomic level (Desai et al, 2010).

Part of the temporal variations and ethnic differential in cancer survival may be due to data issues. For example, lung cancer survival in South Asians, very high in 1986-1994, decreased dramatically by $11 \%$ at 5 years. In 1986-1995, an extra 8\% of South Asians compared with non-South Asians were excluded from the analysis, while the difference between both ethnic groups decreased to 3\% in 1996-2004 (data available on request). Most of this difference was seen in the exclusion of cases due to unknown vital status, that is, patients could not be traced to assess their vital status. This could reflect a migration effect because of the South Asian patients returning to their home country to die (Pablos-Mendez, 1994, Scott and Timaeus, 2013). Restricting the age range to patients diagnosed between 15 and 69 years of age has reduced this effect, although the proportion of unknown vital status was relatively constant in all age groups (data not shown). Furthermore, in order to evaluate whether the proportion of patients wrongly recorded as alive (also called 'immortals') at the time of the analysis differed by ethnicity, we calculated (on the full cohort) the proportions of patients who appeared to have survived beyond their 105th birthday by ethnicity: they were fairly similar. We also looked at the long-term survivors for bad prognosis cancers. For liver cancer, $5.7 \%$ of South Asians survived beyond 10 years (5.4\% in ages 15-69) compared with $0.9 \%(0.7 \%$ in ages $15-69)$ in non-South Asians; for lung cancer, 9\% (7.6\% in ages $15-69)$ of South Asians and $1.7 \%$ (1.4\% in ages 15-69) of non-South Asians. It suggests some differential losses to follow-up (death) by ethnicity.

During the study period, the widest changes in ethnicity-specific proportion of patients excluded were for liver, lung, and ovarian cancers and for NHL, whereas survival in South Asians either became worse than in non-South Asians (NHL) or dropped dramatically (lung). Except for liver cancer, the proportions of patients excluded in the most recent period were not greater than $3 \%$ of the cases. It suggests that the more recent comparisons in cancer survival between both ethnic groups can be considered as more reliable.

Screening uptake in South Asians was lower than in non-South Asians in 1996 (Szczepura et al, 2008), which might be reflected in the slower increase in 5-year survival from breast cancer in South Asians. Similarly, the situation for prostate cancer may be comparable with lower use of PSA (Melia et al, 2004). Policy on implementation of the mass screening programme for colorectal cancer (introduced since 2006) should account for differential screening uptake in South Asians. However, it should be noted that two-thirds of South Asian cancer patients belongs to the two most deprived groups. As screening uptake decreases with increasing deprivation, disentangling the role of ethnicity from that of deprivation remains challenging. Nevertheless, differential trends in survival between both ethnic groups could be partly due to the differential access to early diagnosis in the more recent years. Stage-specific survival was not performed because of lack of good quality information on tumour stage in the national cancer registry for the whole study period.

Cancer beliefs and attitudes vary between countries and, within a country, between ethnic groups (Forbes et al, 2011, 2013). South Asian women were found to have lower breast cancer awareness than White women (Forbes et al, 2011), while a qualitative study concluded that South Asian beliefs hindered their understanding of and access to breast cancer screening (Karbani et al, 2011). Younger generations, up to a third of the cancer patients in the most recent years of our study, may be more aware and receptive to public health messages but communications targeted to minority ethnic groups should still be used to reach newer generations and counteract misplaced beliefs. It is worth noticing that incidence of cervical cancer, although higher in South Asians than in non-South Asians, followed a similar downward trend in both ethnic groups (data not shown). That may reflect comparable trends in prevalence of risk factors and screening uptake. Two studies showed a survival advantage of South Asians for breast cancer in 
the late 1980s and early 1990s (dos Santos Silva et al, 2003; Farooq and Coleman, 2005). Breast cancer survival in non-South Asians is now similar to that in South Asians, and encouraging selfexamination and screening among South Asians should be a priority to maintain high South Asian survival.

Area-based deprivation measures were allocated to each cancer patient and split into five categories according to the quintiles of these deprivation measures in the English population. If this distribution remains fairly uniform in White patients, nearly twothirds of the South Asian population and cancer patients fall into the two most deprived categories (data not shown). The deprivation gradient in cancer incidence was much less steep in South Asians than among non-South Asians (Maringe et al, 2013). By contrast, we found that deprivation gradient in cancer survival was generally similar in both ethnic groups. Socioeconomic inequalities in cancer survival did not decline following the implementation of the NHS Cancer Plan (Rachet et al, 2010), in particular as a consequence of a more rapid increase in survival among the more affluent cancer patients. Because of the skewed distribution towards more deprived categories of the South Asians, the slower survival increase in this population reported here could reflect more generally the experience of the most deprived groups in the population.

The more recent patterns of cancer survival in South Asians look rather similar to those for non-South Asians in England. The protective effect of South Asian ethnicity on cancer incidence and survival seems to have faded. This may be explained by the fact that, in addition to data quality issues, both the first and subsequent generations age and become more westernised. Majority of South Asian population, however, lives in most deprived areas while, in England, poorer cancer survival is largely driven by deprivation (Rachet et al, 2010). Deprivation is also associated with low screening uptake (von Wagner et al, 2009) and sub-optimal cancer management (Morris et al, 2008; Lejeune et al, 2010; Rachet et al, 2010). South Asians show slow increase in breast and prostate cancer survival; both cancers are associated with either a screening programme or a test for early stage disease. Without appropriate action, at least on deprivation-related factors, deficits in cancer survival may appear among South Asians in the near future.

\section{ACKNOWLEDGEMENTS}

CM participated in this research as part of the work of The Policy Research Unit in Cancer Awareness, Screening and Early Diagnosis. The Policy Research Unit in Cancer Awareness, Screening and Early Diagnosis receives funding for a research programme from the Department of Health Policy Research Programme. It is a collaboration between researchers from seven institutions (Queen Mary University of London, UCL, King's College London, London School of Hygiene and Tropical Medicine, Hull York Medical School, Durham University and Peninsula Medical School). This work was also partly based on preliminary work funded by a Cancer Research UK project grant (C23112/A2810, PI: PM). RL and BR were supported by the Cancer Research UK Cancer Survival Programme grant C1336/A11700.

\section{CONFLICT OF INTEREST}

The authors declare no conflict of interest.

\section{REFERENCES}

Ali R, Barnes I, Kan SW, Beral V (2010) Cancer incidence in British Indians and British whites in Leicester, 2001-2006. Br J Cancer 103: 143-148.
Ash M, Robinson DE (2009) Inequality, race, and mortality in U.S. cities: a political and econometric review of Deaton and Lubotsky $(56: 6,1139$ 1153, 2003). Soc Sci Med 68: 1909-1913.

Aspinall PJ, Jacobson B (2007) Why poor quality of ethnicity data should not preclude its use for identifying disparities in health and healthcare. Qual Saf Health Care 16: 176-180.

Bassett MT, Kreiger N (1986) Social class and black-white differences in breast cancer survival. Am J Public Health 76: 1400-1403.

Bates JH, Hofer BM, Parikh-Patel A (2008) Cervical cancer incidence, mortality, and survival among Asian subgroups in California, 1990-2004. Cancer 113: 2955-2963.

Breen N, Wesley MN, Merrill RM, Johnson K (1999) The relationship of socio-economic status and access to minimum expected therapy among female breast cancer patients in the National Cancer Institute Black-White cancer survival study. Ethn Dis 9: 111-125.

Cancer Research UK Cancer Survival Group (2004) Life tables for England and Wales by sex, calendar period, region and deprivation [Online]. London School of Hygiene \& Tropical Medicine. Available at http:// www.lshtm.ac.uk/eph/ncde/cancersurvival/tools/index.html.

Carstairs V (1995) Deprivation indices: their interpretation and use in relation to health. J Epidemiol Community Health 49(Suppl 2): 3-8.

Clerc-Urmès I, Grzebyk M, Hédelin G (2014) Net survival estimation with stns. Stata J 14: 87-102.

Corazziari I, Quinn MJ, Capocaccia R (2004) Standard cancer patient population for age standardising survival ratios. Eur J Cancer 40: 2307-2316.

Department of the Environment Transport and the Regions (2000) Measuring Multiple Deprivation at the Small Area Level: the Indices of Deprivation 2000. DETR: London.

Desai M, Rachet B, Coleman MP, McKee M (2010) Two countries divided by a common language: health systems in the UK and USA. J R Soc Med 103 : 283-287.

dos Santos Silva I, Mangtani P, De Stavola BL, Bell CMJ, Quinn MJ, Mayer D (2003) Survival from breast cancer among South Asian and non-South Asian women resident in South East England. Br J Cancer 89: 508-512.

Downing A, West RM, Gilthorpe MS, Lawrence G, Forman D (2011) Using routinely collected health data to investigate the association between ethnicity and breast cancer incidence and survival: what is the impact of missing data and multiple ethnicities? Ethn Health 16: 201-212.

Farooq S, Coleman MP (2005) Breast cancer survival in South Asian women in England and Wales. J Epidemiol Community Health 59: 402-406.

Forbes LJ, Atkins L, Thurnham A, Layburn J, Haste F, Ramirez AJ (2011) Breast cancer awareness and barriers to symptomatic presentation among women from different ethnic groups in East London. Br J Cancer 105: 1474-1479.

Forbes LJ, Simon AE, Warburton F, Boniface D, Brain KE, Dessaix A, Donnelly C, Haynes K, Hvidberg L, Lagerlund M, Lockwood G, Tishelman C, Vedsted P, Vigmostad MN, Ramirez AJ, Wardle J (2013) Differences in cancer awareness and beliefs between Australia, Canada, Denmark, Norway, Sweden and the UK (the International Cancer Benchmarking Partnership): do they contribute to differences in cancer survival? Br J Cancer 108: 292-300.

Gomez SL, Clarke CA, Shema SJ, Chang ET, Keegan TH, Glaser SL (2010) Disparities in breast cancer survival among Asian women by ethnicity and immigrant status: a population-based study. Am J Public Health 100: 861-869.

Jack RH, Davies EA, Møller H (2009) Breast cancer incidence, stage, treatment and survival in ethnic groups in South East England. Br J Cancer 100: 545-550.

Karbani G, Lim JN, Hewison J, Atkin K, Horgan K, Lansdown M, Chu CE (2011) Culture, attitude and knowledge about breast cancer and preventive measures: a qualitative study of South Asian breast cancer patients in the UK. Asian Pac J Cancer Prev 12: 1619-1626.

Lambert PC, Royston P (2009) Further development of flexible parametric models for survival analysis. Stata J 9: 265-290.

Lannin DR, Mathews HF, Mitchell J, Swanson MS, Swanson FH, Edwards MS (1998) Influence of socioeconomic and cultural factors on racial differences in late-stage presentation of breast cancer. JAMA 279: 1801-1807.

Lejeune C, Sassi F, Ellis L, Godward S, Mak V, Day M, Rachet B (2010) Socioeconomic disparities in access to treatment and their impact on colorectal cancer survival. Int J Epidemiol 39: 710-717.

Li R, Abela L, Moore J, Woods LM, Nur U, Rachet B, Allemani C, Coleman MP (2014) Control of data quality for population-based cancer survival analysis. Cancer Epidemiol 38: 314-320.

Mangtani P, Maringe C, Rachet B, Coleman MP, dos Santos Silva I (2010) Cancer mortality in ethnic South Asian migrants in England and Wales (1993-2003): patterns in the overall population and in first and subsequent generations. Br J Cancer 102: 1438-1443. 
Maringe C, Mangtani M, Rachet B, Leon DA, Coleman MP, dos Santos Silva I (2013) Cancer incidence in South Asian migrants to England, 1986-2004: unravelling ethnic from socio-economic differentials. Int J Cancer 132: 1886-1894.

Melia J, Moss S, Johns L. Contributors in the participating laboratories (2004) Rates of prostate-specific antigen testing in general practice in England and Wales in asymptomatic and symptomatic patients: a cross-sectional study. BJU Int 94: 51-56.

Moles DR, Fedele S, Speight PM, Porter SR (2007) The unclear role of ethnicity in health inequalities: the scenario of oral cancer incidence and survival in the British South Asian population. Oral Oncol 43: 831-834.

Morris EJA, Quirke P, Thomas JD, Fairley L, Cottier B, Forman D (2008) Unacceptable variation in abdominoperineal excision rates for rectal cancer: time to intervene? Gut 57: 1690-1697.

Nanchahal K, Mangtani P, Alston M, dos Santos Silva I (2001) Development and validation of a computerized South Asian names and Group Recognition Algorithm (SANGRA) for use in British health-related studies. J Public Health Med 23: 278-285.

National Cancer Intelligence Network (2009) Cancer Incidence and Survival by Major Ethnic Group, England, 2002-2006. NCIN: London.

Nelson CP, Lambert PC, Squire IB, Jones DR (2007) Flexible parametric models for relative survival, with application in coronary heart disease. Stat Med 26: 5486-5498.

Office for National Statistics (2003) Census 2001 homepage [Online]. Available at www.statistics.gov.uk/census2001/.

Ortiz-Ortiz KJ, Ramirez-Garcia R, Cruz-Correa M, Rios-Gonzalez MY, Ortiz AP (2014) Effects of type of health insurance coverage on colorectal cancer survival in puerto rico: a population-based study. PLoS One 9: e96746.

Ou SH, Ziogas A, Zell JA (2009) Asian ethnicity is a favorable prognostic factor for overall survival in non-small cell lung cancer (NSCLC) and is independent of smoking status. J Thorac Oncol 4: 1083-1093.

Pablos-Mendez A (1994) Mortality Among Hispanics. JAMA 271: 1237-1238.

Parikh-Patel A, Mills PK, Jain RV (2006) Breast cancer survival among South Asian women in California (United States). Cancer Causes Control 17: $267-272$.

Pineda MD, White E, Kristal AR, Taylor V (2001) Asian breast cancer survival in the US: a comparison between Asian immigrants, US-born Asian Americans and Caucasians. Int J Epidemiol 30: 976-982.

Pohar Perme M, Stare J, Estève J (2012) On estimation in relative survival. Biometrics 68: 113-120.

Rachet B, Ellis L, Maringe C, Nur U, Chu T, Quaresma M, Shah A, Walters S, Woods LM, Forman D, Coleman MP (2010) Socioeconomic inequalities in cancer survival in England after the NHS Cancer Plan. Br J Cancer 103: 446-453.

Robbins AS, Koppie TM, Gomez SL, Parikh-Patel A, Mills PK (2007) Differences in prognostic factors and survival among white and Asian men with prostate cancer, California, 1995-2004. Cancer 110: 1255-1263.

Scott AP, Timaeus IM (2013) Mortality differentials 1991-2005 by selfreported ethnicity: findings from the ONS Longitudinal Study. J Epidemiol Community Health 67: 743-750.

Smith LK, Botha JL, Benghiat A, Steward WP (2003) Latest trends in cancer incidence among UK South Asians in Leicester. Br J Cancer 89: $70-73$.

StataCorp (2013) STATA Statistical Software. 13 edn. Stata Corporation: College Station, TX, USA.

Szczepura A, Price C, Gumber A (2008) Breast and bowel cancer screening uptake patterns over 15 years for UK south Asian ethnic minority populations, corrected for differences in socio-demographic characteristics. BMC Public Health 8: 346.

Tehranifar P, Neugut AI, Phelan JC, Link BG, Liao Y, Desai M, Terry MB (2009) Medical advances and racial/ethnic disparities in cancer survival. Cancer Epidemiol Biomarkers Prev 18: 2701-2708.

van Laar M, McKinney PA, Stark DP, Glaser A, Kinsey SE, Lewis IJ, Picton SV, Richards M, Norman PD, Feltbower RG (2012) Survival trends of cancer amongst the south Asian and non-south Asian population under 30 years of age in Yorkshire, UK. Cancer Epidemiol 36: e13-e18.

von Wagner C, Good A, Wright D, Rachet B, Obichere A, Bloom S, Wardle J (2009) Inequalities in colorectal cancer screening participation: a neighbourhood level analysis of London areas. Br J Cancer 101: S60-S63.

Warnakulasuriya KAAS, Johnson NW, Linklater KM, Bell J (1999) Cancer of mouth, pharynx and nasopharynx in Asian and Chinese immigrants resident in Thames regions. Oral Oncol 35: 471-475.

Winter H, Cheng KK, Cummins C, Maric R, Silcocks P, Varghese C (1999) Cancer incidence in the south Asian population of England (1990-92). Br J Cancer 79: 645-654.

(c) (1) (2)(2) This work is licensed under the Creative Commons ay we sa Attribution-Non-Commercial-Share Alike 4.0 International License. To view a copy of this license, visit http:// creativecommons.org/licenses/by-nc-sa/4.0/

Supplementary Information accompanies this paper on British Journal of Cancer website (http://www.nature.com/bjc) 